\title{
Pulsar shapes explained at last
}

\author{
A new classification of the shapes of radio pulses from pulsating stars is a reminder that some matters \\ remain unresolved even after two decades of energetic research.
}

THE temptation to forget that nearly 21 years have passed since the discovery of pulsating stars (see Nature 217, 709; 1968) can only be reinforced by the simple truth that people in the field are still hesitant of being dogmatic about the mechanism of radio emission from these objects. All this is curiously in contrast with what seemed the helter-skelter deepening of understanding that followed the first discovery.

Then, as this journal knows all too well, people were talking of emission from dwarf or even neutron stars within weeks of the discovery of the first pulsar at Cambridge. Professor Thomas Gold was the first to spell out the doctrine in detail. Within a year, there was evidence from timing observations that the spin of some neutron stars could unpredictably accelerate, presumably as a consequence of the rearrangement of solid material on the surface. The idea that spinning neutron stars will usually be magnetized, with surface field strengths unimaginably greater than those available on the surface of the Earth, was also an easy step forward, accounting among other things for the mostly uniform deceleration of the spin of a pulsar. Yet authors in the field have seemingly been hard-pressed to account for the shapes of the radio pulses observed on the surface of the Earth.

Much of the difficulty is diversity: The shape of the pulse is the variation of the radio-emission intensity as a function of phase in the repetitive period of the pulsar. Historically, it fell to people at Jodrell Bank, the radioastronomy observatory at the University of Manchester, to produce the first pulse shapes. To begin with, as even now, they were surprising and even puzzling. While the pulses from some rotating neutron stars are what might be expected - simple gaussian-like excesses of radio-emission - others were double peaks concentrated in one part of the duty cycle while others were still more confused, with a small 'inter-pulse pulse' somewhere between the main emissions. To begin with, this variety of shapes seemed to offer a means of explaining what was happening at the surface of a pulsating star, but the diversity was such that people were reduced to attempts merely at classification.

Only now, after twenty years, does the process of classification seem to make a coherent tale. Appropriately, one of those responsible is A.G. Lyne from Jodrell Bank, one of the group that discovered the second known pulsar. With R. N. Manchester, another pulsar observer of long standing now at the CSIRO Division of Radiophysics at Epping (near Sydney) in Australia, he has now produced the most detailed classification yet of the shapes of pulsar pulses (Mon. Not. R. astr. Soc. 234, 477; 1988) based on analysis of the pulses from more than 200 pulsars.

The essence of the case is simple enough. A spinning neutron star produces a cone-shaped pattern of radio-emission whose axis coincides with the magnetic dipole axis of the neutron star. The magnetic and rotational axes of the star do not necessarily coincide, which means that the cone-shaped pattern of radiation is swept across the sky at the frequency of the rotation of the star; the often-quoted simile of the lighthouse-beam applies. Sharp single pulses are then observed either when the cone is extremely narrow and uniformly filled with radiation or alternatively, when the line of sight from an observer on the surface of the Earth to the magnetic pole of the pulsating star just happens to graze the edge of the cone.

On this simple but correct picture, radio pulses with two peaks arise because the distribution of radio-emission with the revolving cone is far from uniform, and in particular is depleted along the magnetic axis relatively to larger angles with it. Then the two peaks arise as the line of sight intercepts first one direction on the revolving cone and then another, perhaps a whole diameter away.

With the passage of twenty years, there is now a wealth of detailed information with which to work. Lyne and Manchester use, in particular, two kinds of information not available in the early days measurements of the polarization of the radio-emission (gathered as a by-product in attempts to estimate interstellar electron densities from the Faraday rotation of the plane of polarization) and the differences between the pulse profiles at different radio frequencies.

The conclusions are gratifying in that they rationalize diversity, allowing different and complicated pulse shapes to be reconciled with a simple underlying model. The notion that a cone of emission is regularly swept across the sky with the rotational frequency of the underlying neutron star is familiar. So too is the much earlier supposition that the marked separation of some double peaks suggests that the conical pattern of radio-emission from a magnetic pole of the underlying star is often concentrated towards the outer boundaries of the cone.

Surprisingly, given that the mismatch between the rotational and magnetic axes of the star is plainly asymmetric, there is no evidence from the observations that the cone is anything but circular. And the opening angle of the cone appears to be strictly a function of the pulsar period the more rapid the rotation, the wider the cone. Alternatively, the angle of the cone is inversely proportional to the cube root of the pulsar period.

In the pulsars with a marked concentration of radio-emission towards the outer parts of the cone, it seems that there is very little regularity of the distribution of radio-emission within the extremities. Instead, as Lyne and Manchester put it, there are patches of radiation, apparently stable enough to be measured over a great many duty cycles. And while there may be apparently chaotic variations of radioemission within the opening angle of the revolving cone, Lyne and Manchester see no reason to suppose that the mechanisms of radio emission from the two regions are different

In all this, perhaps the most surprising feature of the shape of pulsar pulses is that the emission usually derives from just one of the two magnetic poles. That, after all, is why pulsating stars with inter-pulse pulses are the exceptions, not the rule. In the event, it seems, the second pole is active only in the youngest pulsating stars, either those newly formed in supernova explosions or those which have been newly given a high rotation speed by the accretion of material from a companion star - now the favourite explanation of the origin of the pulsars with rotational periods measured in milliseconds.

Lyne and Manchester thus seem to have brought the classification of pulsar shapes to a satisfactory conclusion. But there is more than mere stamp collecting in the endeavour, for the relationship between the opening-angle of the emission cone and the rotational period of a pulsar is a practical guide to the chance that a particular neutron star will be detected on the surface of the Earth. For the greater the angle, the greater the chance that its cone will sweep across the Earth, and therefore the smaller the chance that observation will be biased against its detection.

John Maddox 